\title{
Soluble starch and composite starch Bioactive Glass 45S5 particles: Synthesis, bioactivity, and interaction with rat bone marrow cells
}

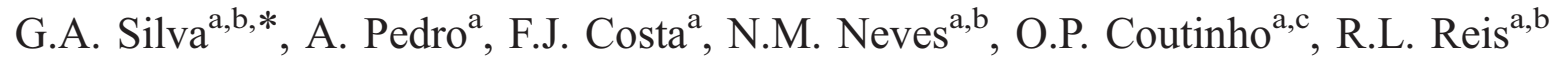 \\ a3B's Research Group-Biomaterials, Biodegradables, Biomimetics, University of Minho, Campus de Gualtar, Braga 4710-057, Portugal \\ ${ }^{\mathrm{b}}$ Department of Polymer Engineering, University of Minho, Campus de Azurém, Guimarães 4800-058, Portugal \\ ${ }^{\mathrm{c}}$ Department of Biology, University of Minho, Campus de Gualtar, Braga 4710-057, Portugal
}

\begin{abstract}
For many biomedical applications, biodegradable and simultaneously bioactive materials are desired. These materials should at the same time be able to support cell function and co-exist with the organism without triggering a relevant immune response.

In this work, the synthesis as well as the bioactivity evaluation of newly developed polymer soluble potato starch and composite (with Bioactive Glass 45S5) micron-size particles are reported. An extremely interesting result is that although with different properties, both polymer and composite particles were able to form a calcium phosphate layer at their surface, which is a clear indication of their bioactivity.

The cytotoxicity and the ability to support cell attachment and growth of the developed materials were also studied, and both polymer and composite materials were shown to be non-cytotoxic. Preliminary results show that both types of materials were found to allow rat bone marrow cells to attach and to proliferate on their surface and to express osteogenic markers, such as alkaline phosphatase and osteopontin.

The obtained results indicate that the developed carriers might be used as substrates for cell culture in vitro, in order to form constructs that might be used as a part of a tissue engineering strategy.
\end{abstract}

(C) 2005 Elsevier B.V. All rights reserved.

Keywords: Starch-based; Bioactive glass; Rat bone marrow cells; Bioactivity; Ca-P layer; Cytotoxicity

\section{Introduction}

Materials for bone repair and regeneration are one of the most studied subjects in the biomaterials field. The particular properties of bone render it difficult to achieve a satisfactory material that gathers all the desired characteristics including among others mechanical properties, bone bonding ability, and biocompatibility. In several applications, biodegradable materials are specially desired, particularly in nonload-bearing applications, where biomaterials used as a bone substitute should be a temporary material serving as a scaffold for bone remodelling [1-3]. This type of material must degrade in a controlled fashion into non-

\footnotetext{
* Corresponding author: Tel.: +351 253604 781/2; fax: +351 253604 492.

E-mail address: gsilva@dep.uminho.pt (G.A. Silva).
}

toxic products that the body can metabolise or excrete via normal physiological mechanisms $[4,5]$.

In vitro models both in terms of biocompatibility and bioactivity evaluation have proven to be valuable tools for testing and screening candidate biomaterials [1,6-8]. One possible route to in vitro induce bioactivity into polymeric substrates is to use autocatalytic deposition methods and biomimetic routes in order to induce Ca-P layer formation on the surface of the polymers [9-11]. Another strategy is to combine polymers with bioactive materials such as hydroxylapatite and bioactive glasses $[12,13]$.

Since most bone implant materials are implanted into adult bone in direct contact with bone marrow tissue, it is advantageous to use bone marrow cell cultures from adult rats to investigate new implant materials [14-16]. Bone marrow cells have been reported to proliferate and differentiate, to express bone cell markers, and to form mineralised nodules in vitro $[17,18]$. In this work, we have 
used such type of cultures aiming at assessing if rat bone marrow cells were capable of displaying this same behaviour in the presence of starch and starch-bioactive glass microparticles. In this way, by isolating and culturing rat bone marrow cells and placing them in contact with potential biomaterials, it is possible to gain insights on foreseen behaviour of the material in vivo.

\section{Materials and methods}

\subsection{Synthesis of soluble starch and soluble starch Bioactive Glass 45S5 particles}

Paselli II (PaII), a soluble potato starch chemically modified (AVEBE, The Netherlands) was the raw material used. The reinforcement material, Bioactive Glass 45S5 (46.1\% $\mathrm{SiO}_{2}, 24.4 \% \mathrm{Na}_{2} \mathrm{O}, 26.9 \% \mathrm{CaO}, 2.6 \% \mathrm{P}_{2} \mathrm{O}_{5}, \mathrm{~mol} \%$ ) was obtained from MO-SCI (Rolla, MO, USA) and ground before use to a particle size smaller than $16 \mu \mathrm{m}$.

For the synthesis of starch and composite particles, Paselli II starch was dissolved in water together with a crosslinking agent, trisodium trimethaphosphate (TSTP). For the synthesis of the composite particles, $30 \%$ of the polymer weight of BG 45S5 was mixed with the polymer powder at this step. This solution was then placed under constant vigorous stirring with a top stirrer, to which a mixture of light mineral oil and sorbitan monooleate (SPAN 80)-selected as emulsifying agent-was slowly added. After the formation of the emulsion, sodium hydroxide was added dropwise in order to activate the crosslinking reaction, which proceeded for $6 \mathrm{~h}$. The emulsion was then destabilised with a mixture of water: acetic acid (1:1) or with $100 \%$ ethanol (in case of composite particles to prevent BG 45S5 reactions). Produced particles were collected, extensively washed, and then freeze-dried.

\subsection{Morphology}

The synthesized particles were morphologically characterized by light microscopy and scanning electron micros- copy (SEM). The particles were observed and photographed in an optical microscope with an image acquisition system (high-resolution colour video camera and Image-Pro Plus analysis software; Media Cybernetics, Silver Spring, Maryland, USA). Scanning electron microscopy (SEM) analysis was performed on gold-coated samples in a Leica Cambridge S-360 (Cambridge, UK), and energy dispersive spectroscopy (EDS) was performed on carbon-coated samples.

\subsection{In vitro bioactivity evaluation}

PaII and PaII-30\% BG particles were tested in vitro for their bioactivity in a $0.05 \mathrm{M}$ Tris hydroxymethyl aminomethane-buffered solution complemented with electrolytes typical for plasma (TE). The use of this solution was described previously by Kokubo et al. [19]. PaII and PaII$30 \% \mathrm{BG}$ particles were immersed in TE at a ratio of $3 \mathrm{mg}$ / $\mathrm{ml}$ in a $\mathrm{CO}_{2}$ incubator at $37{ }^{\circ} \mathrm{C}$. The vials were placed on an orbital shaker at a rotational speed of $150 \mathrm{rpm}$ for up to 21 days without solution exchange. At designed time points $(1,2,3,7,10,14$, and 21 days), the samples were removed from the shaker, the $\mathrm{pH}$ of the solution measured, and the particles separated from the solution by filtration. The particles were then dried and stored in a desiccator until further analysis. The postimmersion solutions were used to perform chemical analysis, namely for changes in $\mathrm{Ca}, \mathrm{Si}$, and $\mathrm{P}$ concentrations, that were evaluated by ICP-OES (inductively coupled plasma-optical emission spectroscopy).

Further characterization of the immersed particles was performed using Fourier transformed infrared attenuated reflection (FTIR-ATR), scanning electron microscopy/ energy dispersive spectroscopy (SEM/EDS) analysis, and $\mathrm{X}$-ray diffraction (XRD) analysis.

\subsection{Short-term cytotoxicity evaluation}

For cytotoxicity evaluation by indirect contact with the materials, two different tests were performed: MTT, a viability test widely used to assess the cytotoxicity of a potential biomaterial, and total protein quantification.
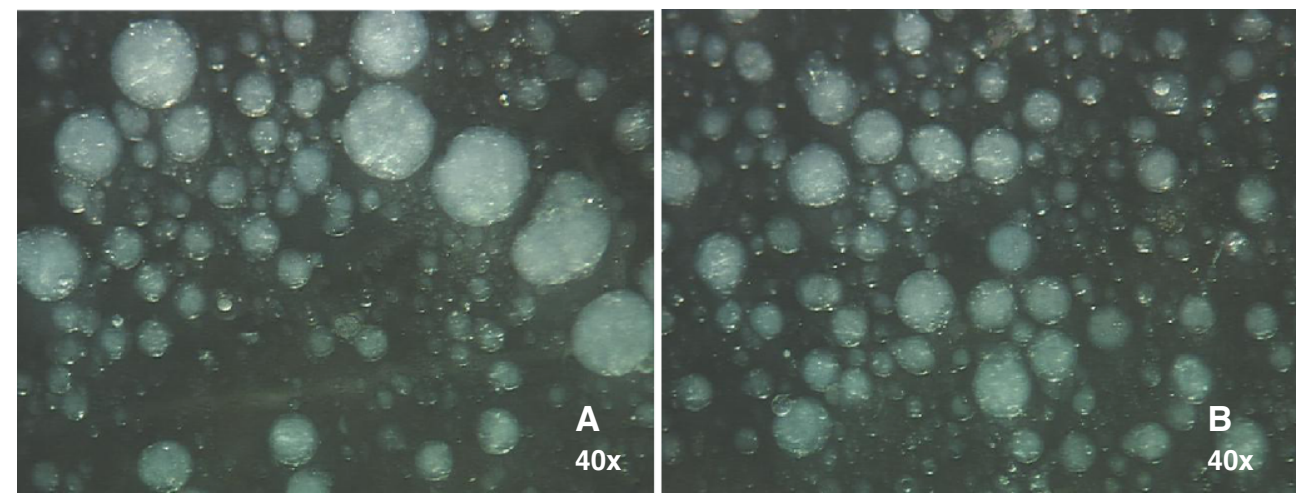

Fig. 1. Light microscopy images of the synthesised particles: PaII (A) and PaII- $30 \%$ BG (B). The appearance of the particles is spherical and no differences in morphology can be attributed to the presence of BG 45S5 (original magnification, $40 \times$ ). 

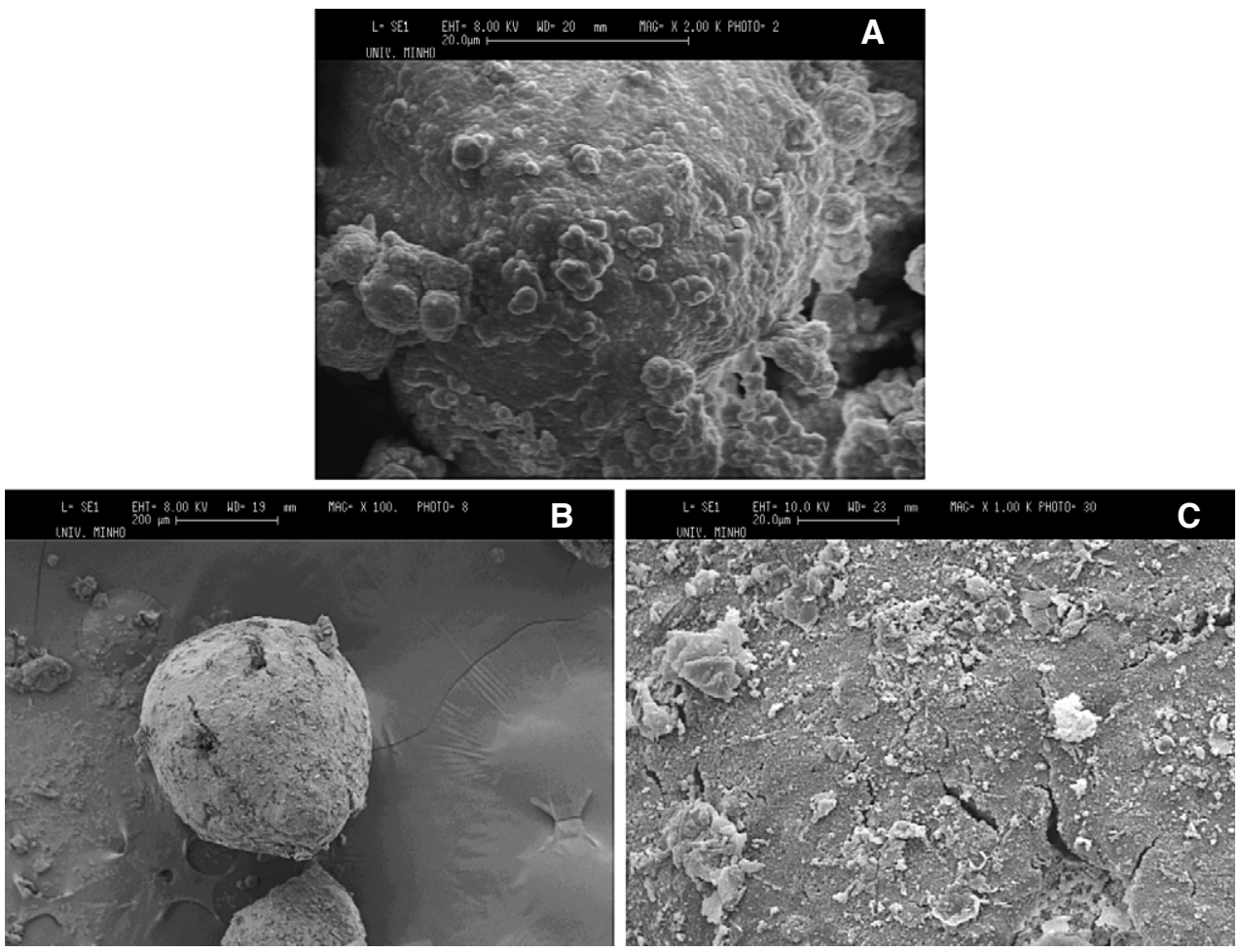

Fig. 2. Representative scanning electron microscope (SEM) images of PaII (A) and PaII-30\% BG particles (B), and surface of composite particles (C).

The cells used in this study were an immortalized cell line of subcutaneous areolar fibroblasts from mouse origin purchased from Collection of Cell Culture (ECACC, UK; ref. 85011425), designated L929. The cells were grown in monolayer systems in Dulbecco's modified Eagle's medium (DMEM) (supplemented with antibiotics and FBS) and used at low passages. The materials were extracted in DMEM culture medium (supplemented as described above) for $24 \mathrm{~h}$ at $60 \mathrm{rpm}$. After this time period, the culture medium with the materials' extracts was filtered and added to the cellular monolayer.
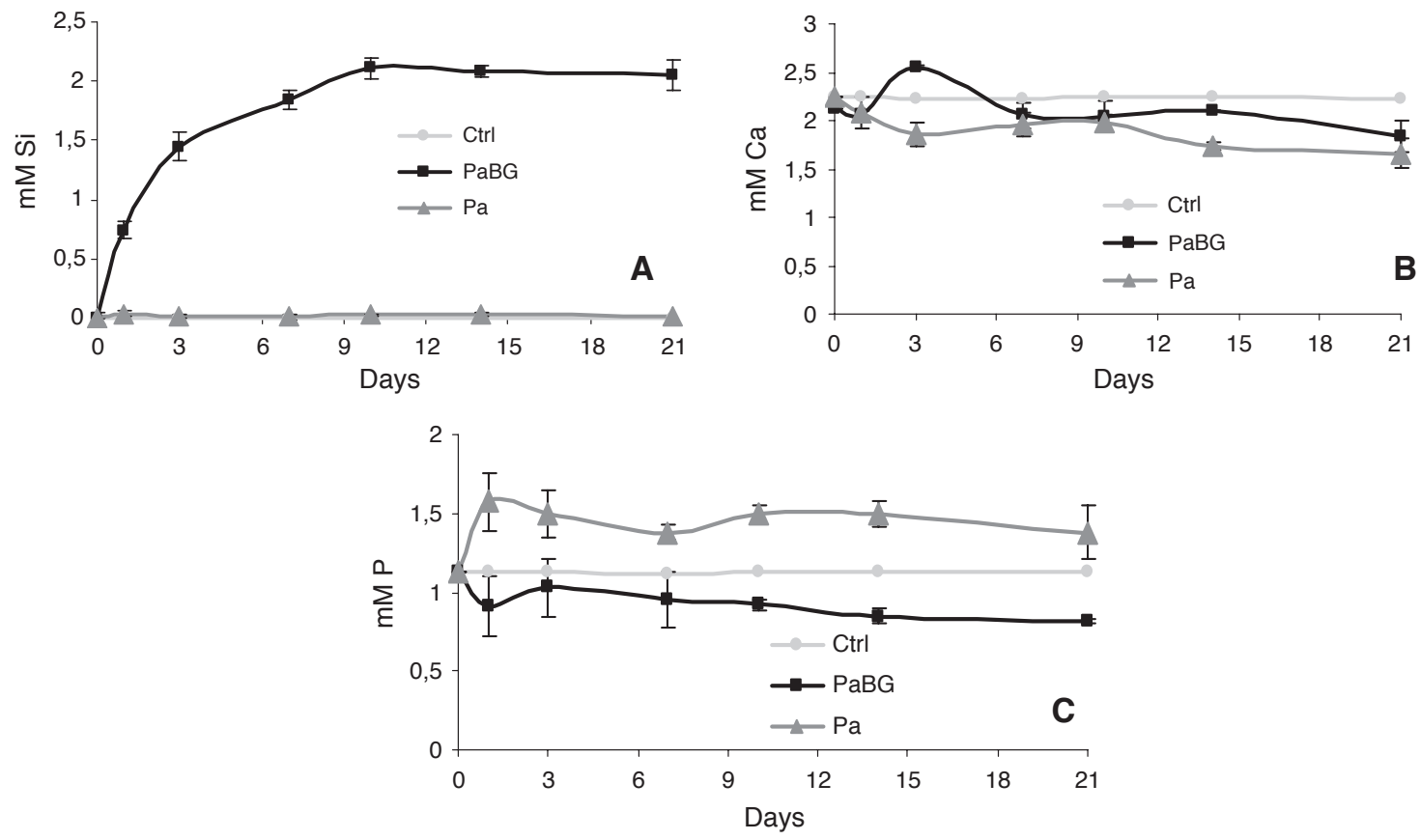

Fig. 3. Si (A), Ca (B), and P (C) profiles during 21 days of immersion in TE (control). Measurements were made on control solutions (Ctrl) and solutions in which either PaII $(\mathrm{Pa})$ or PaII-30\% BG (PaBG) particles were previously immersed. 


$$
\text { Starch-OH }+\mathrm{Na}_{3} \mathrm{P}_{9} \mathrm{O}_{9} \stackrel{\mathrm{NaOH}}{\longrightarrow} \text { Starch-O-P-O-Starch }
$$

Fig. 4. Mechanism of starch crosslinking with trisodium trimethaphosphate in sodium hydroxide aqueous (aq) solution [17].

\subsubsection{MTT}

The MTT test is a biochemical test widely used to assess cytotoxicity by measuring cell viability and proliferation in a qualitative way $[20,21]$. This biochemical test is based in the reduction of (3-(4,5-dimethylthiazol-2-yl)-2,4-diphenyltetrazolium bromide) (which is water-soluble and has a yellow tonality) by the cell mitochondrial enzyme succinate dehydrogenase, yielding a purple colour salt insoluble in water [22]. The salt absorbs at a wavelength of $570 \mathrm{~nm}$ and since only living cells have the capability of metabolising the MTT, it gives a measurement of the viable cells [22]. The procedure followed for this test was as described previously [23]. All the materials were tested in 10 replicates for each extract for at least two independent experiments with reproducible results. The results are expressed as a percentage of the control (scored as 100\% viability) as mean \pm S.E.

\subsubsection{Total protein quantification}

The method that was used to quantify the total protein uses the Micro BCA Protein Assay Reagent Kit, in which bicinchoninic acid (BCA) is the detection reagent for a $\mathrm{Cu}$ complex, which is formed when $\mathrm{Cu}^{2+}$ is reduced by proteins in an alkaline environment [24]. The purple colour product is due to the chelation of two molecules of BCA with one $\mathrm{Cu}$ ion. This complex is water-soluble and absorbs at 562 $\mathrm{nm}$, and its optical density is linearly correlated with protein concentration [24]. The procedure was performed as described in a previous work [23].

All the materials were tested in 10 replicates for each extract for at least three separate experiments, with reproducible results. The results are expressed as percentage of the control (scored as $100 \%$ viability) as mean \pm S.E.

\subsection{Rat bone marrow stromal cells: isolation and culture}

Rat bone marrow cells (RBMC) were isolated and cultured according to the method described by Maniatopoulos et al. [17]. In brief, femora of 5-week-old Wistar rats were removed, cleaned from soft tissue, cut at the epiphyses, and then flushed into $\alpha$-MEM culture medium supplemented with FBS, antibiotics, ascorbic acid, $\beta$-glycerophosphate, and dexamethasone. The cells were maintained at $37{ }^{\circ} \mathrm{C}, 5 \% \mathrm{CO}_{2}$ for 6 days, with medium change for removing debris.

Meanwhile, a pre-determined amount of PaII and PaII$30 \%$ BG particles enough to cover the bottom of a well of a 24-well plate was weighted and placed in non-adherent cell culture plates. The use of this particular type of culture plates is to prevent the quantification of any response coming from cells adhered to the well and not to the materials. Prior to cell seeding, the materials were ethanolsterilised and pre-incubated for $24 \mathrm{~h}$ in $500 \mu \mathrm{l}$ of culture medium in the 24-well plates.

The cells were detached using trypsin and seeded at a density of $5 \times 10^{4}$ cells/well. Two studies were performedboth polymer and composite materials were cultured in two different conditions, with culture medium supplemented with and without dexamethasone. Dexamethasone was used-together with ascorbic acid-as a means of inducing the commitment of rat bone marrow cells into the osteoblastic lineage. The absence of dexamethasone in one set of experiments had the aim of assessing if cells cultured on the surface of starch-based particles could commit to the osteoblastic lineage even in the absence of dexamethasone.

The cultures were maintained for 17 days, with periodic evaluations whenever the culture medium was changed (every 3 days).

\subsection{Total protein quantification and alkaline phosphatase activity}

Total protein quantification was performed as described in Section 2.4.2. In each well, the culture medium was removed (for ALP quantification) and the particles washed
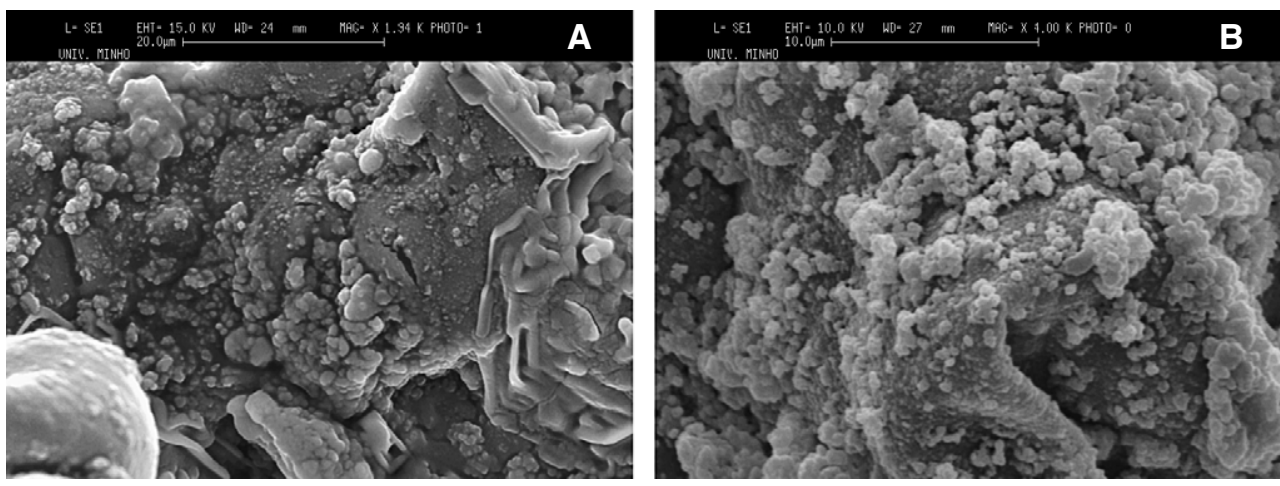

Fig. 5. PaII particles after 21 days of immersion (A and B). Image (B): a higher magnification showing the morphology of the Ca-P layer covering the surface of the particles. 

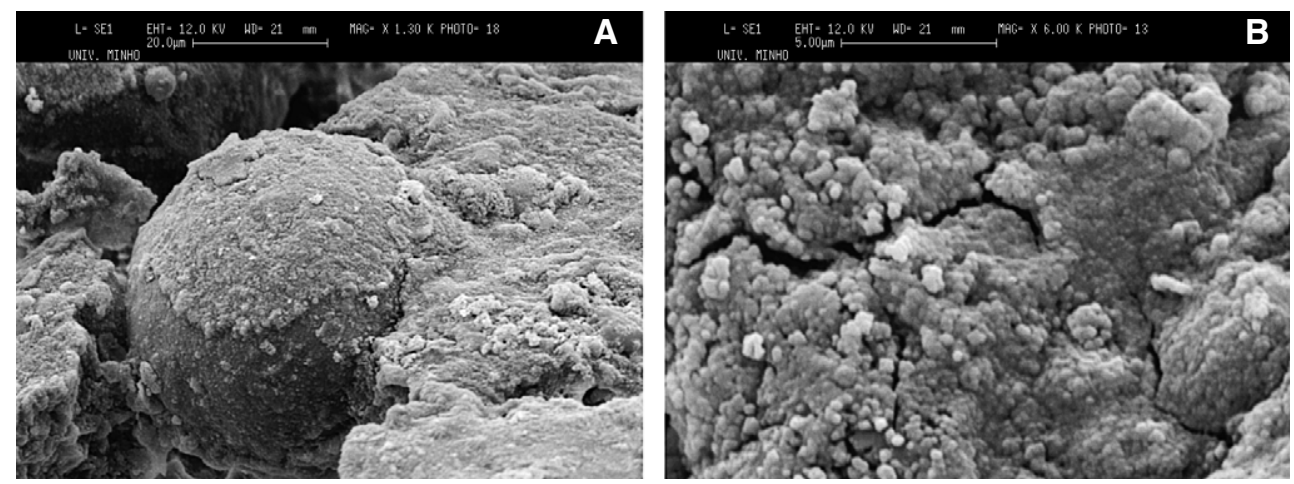

Fig. 6. Pa-30\% BG particles covered with a Ca-P layer after 14 days of immersion (A) and surface close-up after 7 days of immersion (B).

with PBS for total protein quantification. Then $500 \mu \mathrm{l}$ of PBS was added followed by $100 \mu \mathrm{l}$ of the BCA reagent to each well, and further incubated for $2 \mathrm{~h}$. The optical densities were measured and total protein (in $\mu \mathrm{g}$ ) was determined using a BSA standard curve.

Alkaline phosphatase (ALP) activity was assayed by the release of $p$-nitrophenol from $p$-nitrophenylphosphate as described before [25].

\subsection{Immunocytochemistry}

The 14-day samples (control particles and particles cultured with cells) were washed twice with $2 \mathrm{ml}$ of PBS $1 \times$ (phosphate buffered saline tablets; Sigma, St. Louis, USA) for $10 \mathrm{~min}$. The samples were fixated with $0.5 \%$ glutaraldehyde in PBS $1 \times$ (glutaraldehyde grade II 25\% aqs.; Sigma-Aldrich, St. Louis, USA) and incubated for 30 min at $37{ }^{\circ} \mathrm{C}$. After fixation, samples were washed again twice with PBS for $10 \mathrm{~min}$.

Afterwards cells were permeabilised through the incubation with a $0.2 \%$ solution of Triton X-100 in PBS, 2 min at room temperature, followed by a washing step, three times with PBS for $5 \mathrm{~min}$. The primary antibody used was the mouse monoclonal antibody MPIIIB10 (DSHB, University of Iowa, IA, USA) that was raised against a rat bone extract fraction.

Samples were incubated with the primary antibody in a humidified atmosphere at room temperature with a 1/100 dilution (in a 3\% BSA/PBS solution) of the MPIIIB10 antiosteopontin antibody for $1 \mathrm{~h}$. This incubation period was followed by washings $(3 \times)$ with PBS for 5 min each. For detection of the primary antibody, a 1/100 dilution of the secondary antibody conjugated with a fluorochrome (Alexa Fluor ${ }^{\mathrm{TM}} 488$ goat anti-mouse IgG; Molecular Probes, Leiden, The Netherlands) was added to each lamella for 1 $\mathrm{h}$ at room temperature and protected from light. After the secondary antibody incubation, the samples were again washed with PBS $(3 \times, 5$ min each).

The ProLong ${ }^{\circledR}$ Antifade Kit montage medium (ProLong ${ }^{\circledR}$ Antifade Kit (P-7481), Molecular Probes, Leiden, The Netherlands) was prepared and dropped onto the samples and dried for $1 \mathrm{~h}$, after which the samples where observed on a fluorescence microscope with a coupled camera/image acquisition system.

\section{Results and discussion}

\subsection{Synthesis of polymer and composite starch and starch Bioactive Glass $45 S 5$}

The synthesis of soluble starch PaII and PaII-30\% BG 45S5 particles yielded particles with a spherical morphology with varied sizes, up to $500 \mu \mathrm{m}$ (Fig. 1A and B). The presence of $\mathrm{BG} 45 \mathrm{~S} 5$ does not seem to affect both the morphology and the size of the particles (Fig. 1B).
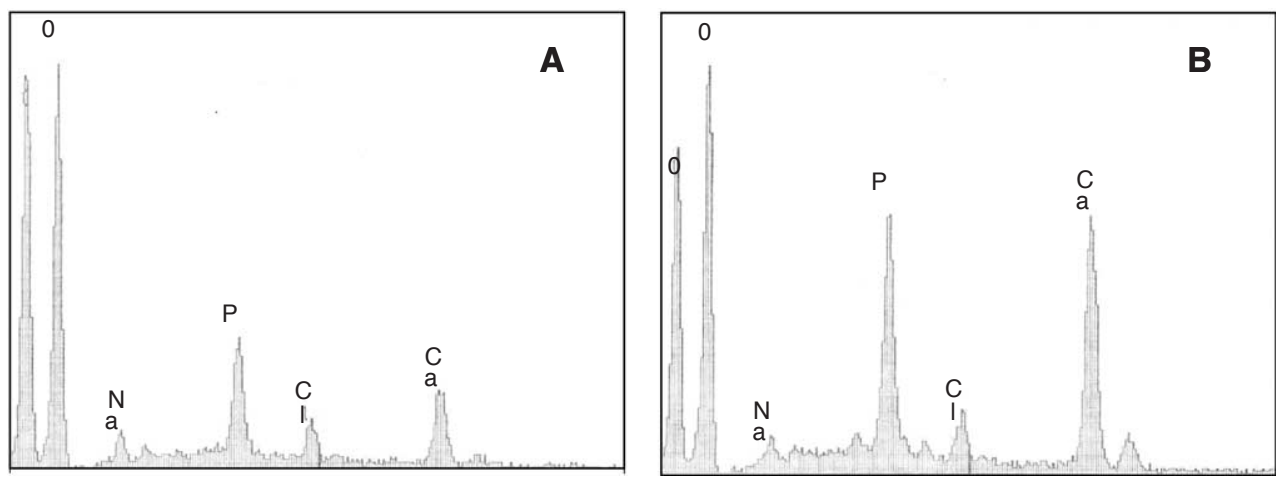

Fig. 7. Energy dispersive X-ray analysis for PaII polymeric particles (A) and PaII-30\% BG 45S5 composite particles (B). 


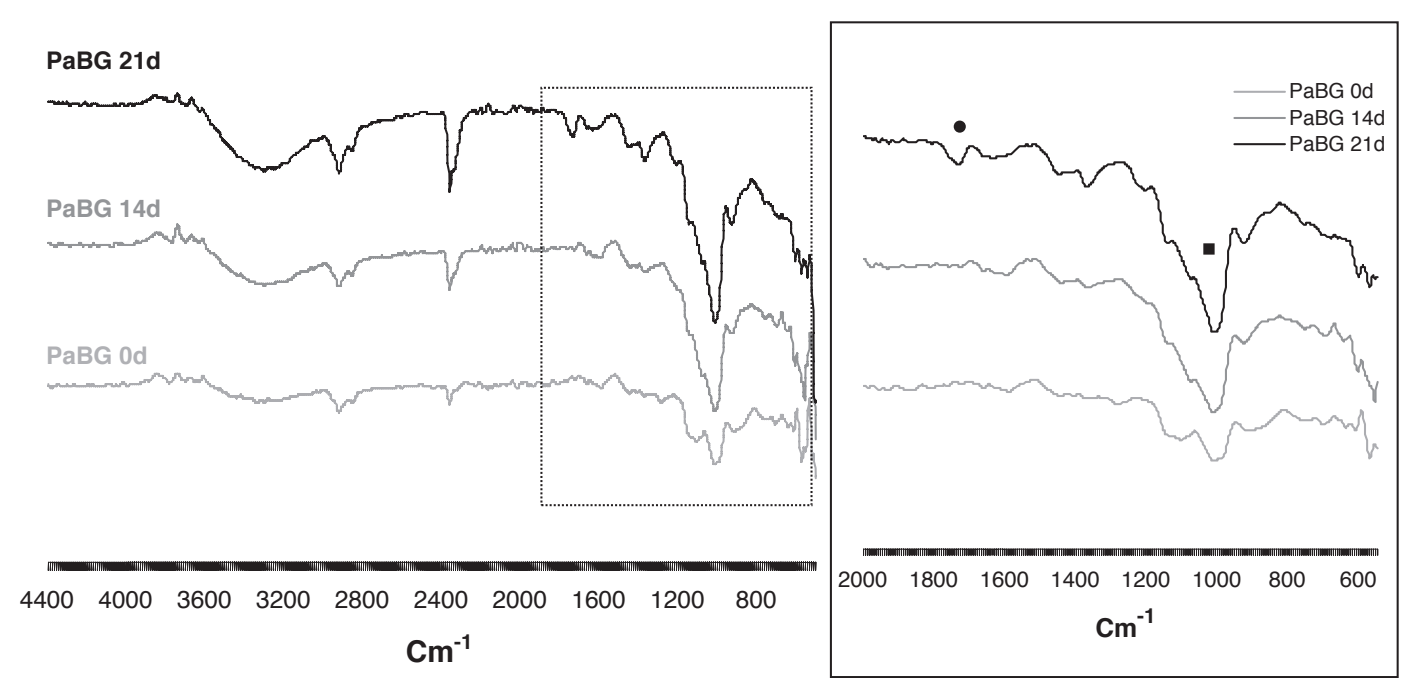

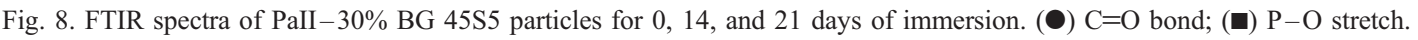

However, scanning electron microscopy analysis (Fig. 2) of both PaII polymer (A) and $\mathrm{Pa}-30 \%$ BG composite (B and $\mathrm{C}$ ) particles shows that at higher magnifications, some differences between the polymer and composite particle surface are evident. Regarding the morphology, composite particles seem to be more regularly spherical than polymer ones. The major differences are observed at the surface of the particles, where polymeric particles seem to be composed by small particles that were crosslinked into a bigger network (Fig. 2A).

As for composite particles (Fig. 2B and C), the surface is smoother compared with the one from polymer (Fig. 2A) particles. Since the synthesis methodology is the same, these differences can be attributed to the presence of BG $45 \mathrm{~S} 5$ in the composite particles.

\subsection{Bioactivity evaluation}

The in vitro bioactivity evaluation was based on immersion of the particles in a fluid simulating the blood plasma ionic composition (Tris buffer complemented with electrolytes) up to 21 days. The results for the chemical analysis performed as function of the immersion time are presented in Fig. 3. Fig. 3A-C display the results of the chemical analysis of $\mathrm{Si}, \mathrm{Ca}$, and $\mathrm{P}$ in immersion solution for the analysed time points.

As can be seen in Fig. 3A, BG 45S5 was successfully incorporated into PaII-30\% BG particles, as seen by the cumulative Si release for the immersion times. As for PaII particles, no Si release was observed, as expected. Together with the $\mathrm{Si}$, also $\mathrm{Ca}$ and $\mathrm{P}$ concentrations in solution were evaluated for both polymer and composite particles. The variation of calcium in the solution (Fig. 3B) is reduced for both PaII and PaII-30\% BG particles, when comparing with the control. This indicates that there is only a minor withdrawal of calcium from the solution. As for phosphate (Fig. 3C), there are differences between the two materials, since for PaII particles, the amount of phosphate in solution increases over time. This phenomenon might be explained by the fact that the synthesis of these materials includes a crosslinking step that uses a phosphate-containing compound, trisodium trimethaphosphate (TSTP), as crosslinking agent. These materials being biodegradable, we might speculate that the increase in phosphate observed in solution might be due to the scission of less strong crosslinked starch chains, thus releasing small starch and phosphate groups into the solution. This phosphate release from the structure of the particles occurs via a mechanism independent of the phosphate precipitation that is occurring

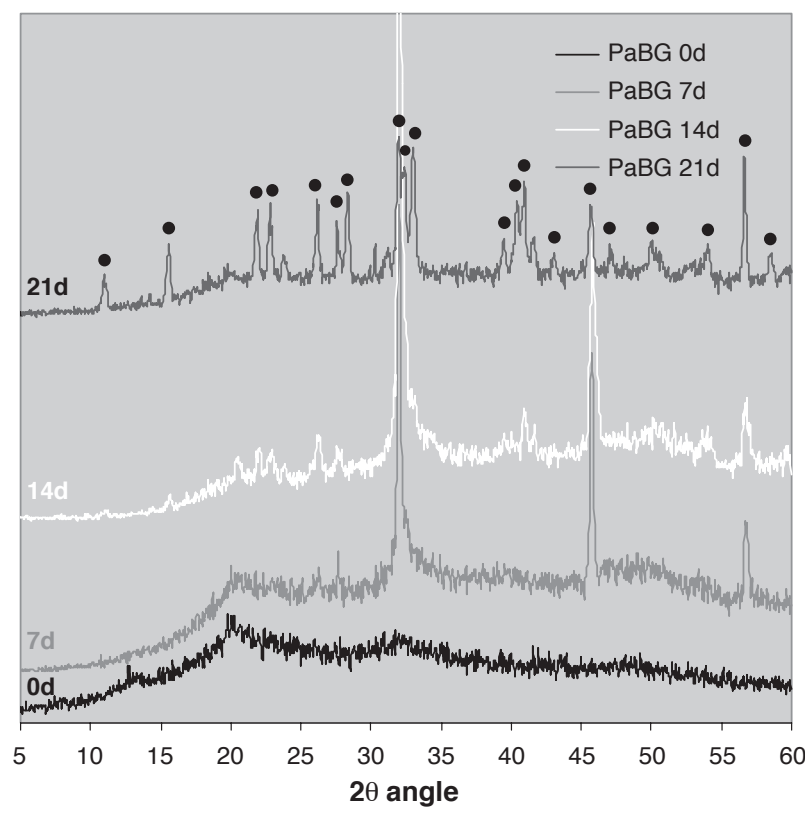

Fig. 9. X-ray dispersion of $\mathrm{Pa}-30 \% \mathrm{BG}$ particles at $0,7,14$, and 21 days of immersion. Main peaks are observed at 26 and 32 at $2 \theta$ angle values, although several secondary peaks are present at other values. The main peaks are typical for HA 90432 JCPDS file. 


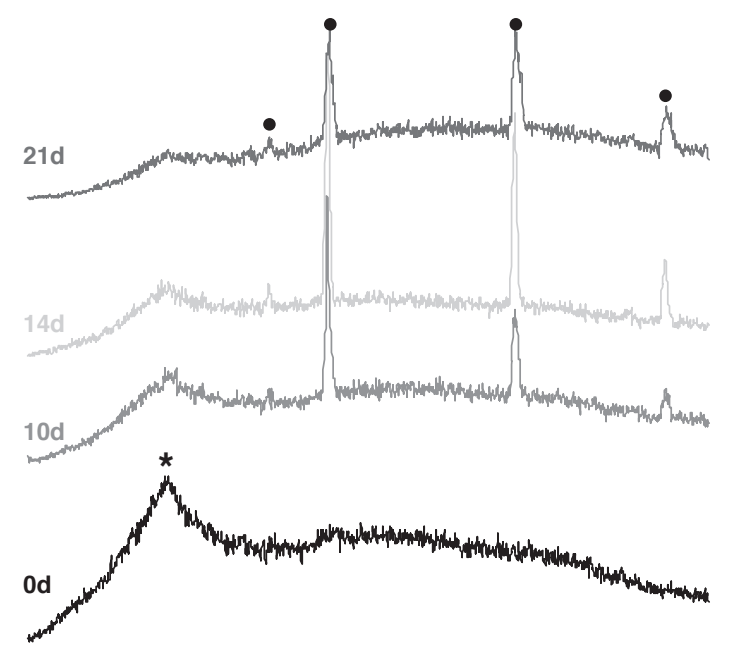

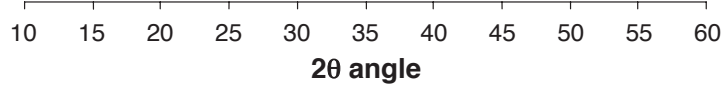

Fig. 10. X-ray dispersion of PaII particles at $0,10,14$, and 21 days of immersion. HA peaks are observed at $32^{\circ}$ and $46^{\circ}$. *Material peak; major peaks appearing at 10,14 , and 21 days of immersion.

at the surface of the particles to form a Ca-P layer. The crosslinking mechanism, as represented in Fig. 4, displays the bonding between the crosslinking agent and starch groups. This phosphate release is most probably higher than the amount of phosphate precipitating at the particles surface, which causes the increase in $\mathrm{P}$ amount in solution observed in the Fig. 3C.

As for composite PaII-30\% BG particles, there is a progressive decrease of phosphate in the solution, indicating that this ion was being precipitated at the surface of the particles, giving origin to a bioactive Ca-P layer (Fig. 3C).

Further analysis by SEM (Figs. 5 and 6) shows that, in fact, both polymer and composite particles display at their surface a deposited Ca-P layer, although more clearly in the composite particles.

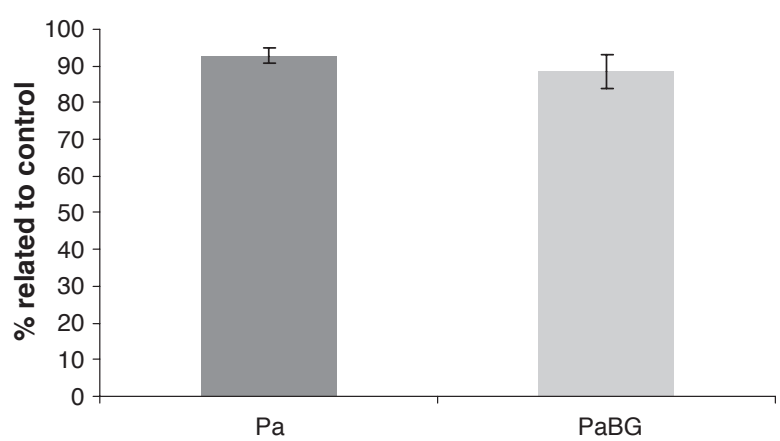

Fig. 11. Total protein quantification for $\mathrm{Pa}$ and $\mathrm{Pa}-30 \% \mathrm{BG} 45 \mathrm{~S} 5$ particles. The values shown are presented as a percentage of the control (cells cultured with culture medium, scored $100 \%$ ) and, for both materials, the values are above $89 \%$. No statistically significant differences are observed between both materials $(p>0.05)$.

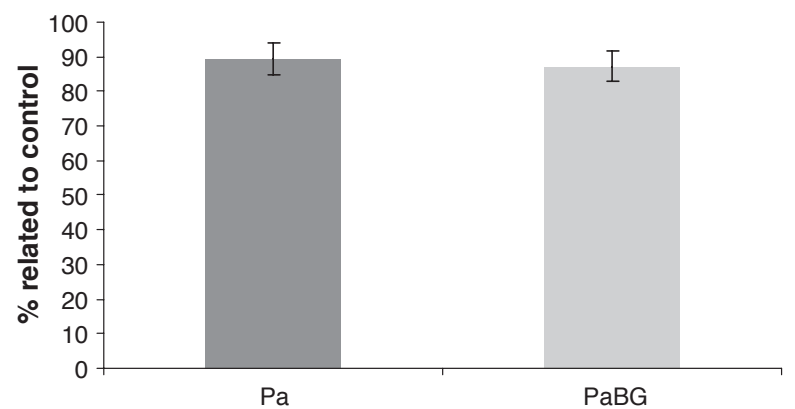

Fig. 12. MTT test for $\mathrm{Pa}$ and $\mathrm{Pa}-30 \% \mathrm{BG} 45 \mathrm{~S} 5$ particles. The values for both materials are presented as a function of the control (cells cultured with culture medium, scored $100 \%$ ). Both materials present values above $87 \%$. No statistically significant differences are observed between both materials $(p>0.05)$.

For PaII particles, there is a layer of Ca-P deposited at the surface, confirmed by EDS (Fig. 7A), and P seems to be present in higher amounts at the particles' surface than $\mathrm{Ca}$. This result can be explained by the presence of $\mathrm{P}$ in the starch network, which does not allow to determine the Ca-P ratio of the layer. Previous works have shown that materials with terminal $\mathrm{P}$ groups are able to form an apatite layer when soaked in SBF without induction time and at a higher growth rate than for other groups [26]. In the present work, the presence of phosphate in the starch particle network might induce the $\mathrm{Ca}$ deposition and consequent $\mathrm{Ca}-\mathrm{P}$ formation at the surface of the particles, since it is known that the apatite induction is facilitated by increasing concentrations of either $\mathrm{Ca}$ or $\mathrm{P}$ [27].

As for the composite particles (Fig. 7B), EDS analysis also reveals the presence of Ca-P layer formation at the

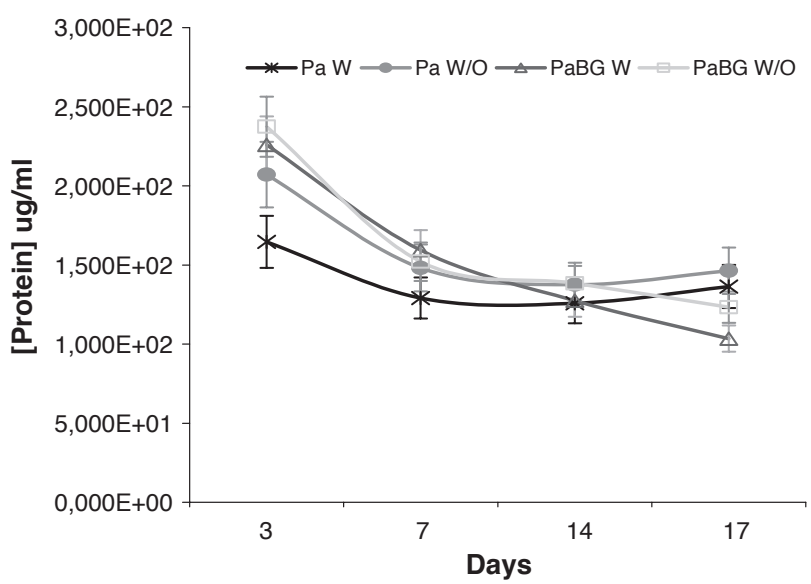

Fig. 13. Total protein content for 17 days of culture. Pa W-polymeric particles cultured with rat bone marrow cells with dexamethasone in culture medium; $\mathrm{Pa} \mathrm{W} / \mathrm{O}$ - polymeric particles cultured with rat bone marrow cells without dexamethasone in culture medium; $\mathrm{PaBG} \mathrm{W}$ - composite particles cultured with rat bone marrow cells with dexamethasone in culture medium; $\mathrm{PaBG} \mathrm{W} / \mathrm{O}$ - composite particles cultured with rat bone marrow cells without dexamethasone in culture medium. 


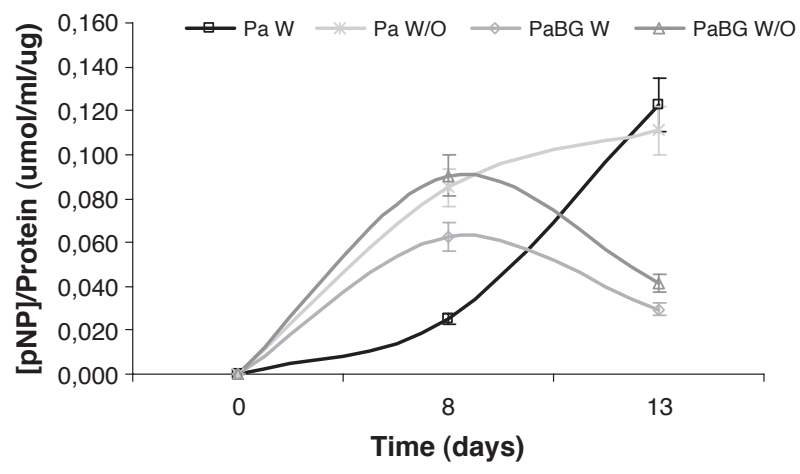

Fig. 14. Alkaline phosphatase activity per protein content. $\mathrm{Pa} \mathrm{W}-$ polymeric particles cultured with rat bone marrow cells with dexamethasone in culture medium; $\mathrm{Pa} \mathrm{W} / \mathrm{O}$ - polymeric particles cultured with rat bone marrow cells without dexamethasone in culture medium; PaBG Wcomposite particles cultured with rat bone marrow cells with dexamethasone in culture medium; $\mathrm{PaBG} \mathrm{W} / \mathrm{O}$ - composite particles cultured with rat bone marrow cells without dexamethasone in culture medium.

surface of the particles, which is already visible at 7 days of immersion (Fig. 6B, SEM image), confirming the stronger bioactive character of the composite particles.

FTIR analysis of PaII-30\% BG 45S5 particles (Fig. 8) reveals differences in the spectra of non-immersed particles ( 0 days of immersion) and after 21 days of immersion. The enhancement of the intensity of bands at $1100-1050 \mathrm{~cm}^{-1}$, which correspond to the $\mathrm{P}-\mathrm{O}$ stretch, as well as of the $\mathrm{C}=\mathrm{O}$ bond at $1740 \mathrm{~cm}^{-1}$, is visible.

The nature of this Ca-P layer regarding its crystallinity by $\mathrm{X}$-ray dispersion, as presented in Fig. 9, was also investigated.

By comparison with the 90432 Hydroxylapatite JCPDS standard, it is possible to observe that $\mathrm{Pa}-30 \% \mathrm{BG}$ particles after 7 days of immersion already present one of the HA main peaks. It is known that the crystallization of insoluble salts involves the formation of metastable precursor phases [26], so with increasing immersion time, the layer of Ca-P is growing and rearranging, and at 14 days, other typical HA peaks are visible that, at 21 days, correspond with a great number of less intense peaks present in the HA 90432 standard.

For PaII particles, the XRD pattern reveals that the layer of Ca-P is growing with time, by the disappearance of the material peak $(*)$ and the appearance of crystalline peaks at $27^{\circ}, 32^{\circ}, 46^{\circ}$, and $57^{\circ}(\bullet)$ (Fig. 10).

\subsection{Short-term cytotoxicity}

The results for the short-term cytotoxicity evaluation are presented in Figs. 11 (total protein) and 12 (MTT). The results are presented as percentages of the control both for MTT and total protein, meaning that the values obtained are in comparison with the $100 \%$ proliferation attributed to the control.

As it can be seen, both polymer and composite materials present total protein values close to the one from the control. The results obtained for both materials are above $89 \%$, which is a good indication of their clearly non-cytotoxic behaviour.

This result is well correlated with the MTT results, in which both materials present viability percentages above $87 \%$ (Fig. 12). Some difference is observed between the results for the polymer and composite materials, and the $t$ test was performed to assess if these differences were significant. The result of the $t$ test shows that no significant differences are found between the two materials $(p>0.05)$.

\subsection{Total protein and ALP quantification}

Fig. 13 displays the evolution of the protein content during 17 days of culture of the particles with rat bone marrow cells.

It is visible that cells are able to adhere to the particle surface, and that protein content reaches a steady state after 7 days of culture, which may indicate that cells are differentiated, since proliferation and differentiation are described as uncoupled processes. Regarding alkaline phosphatase activity, Fig. 14 shows its tendency after normalisation for the total protein content. For composite particles, the ALP highest activity is observed at day 7 of culture with particles; as for polymeric particles, this peak is reached after 14 days of culture. This may indicate that the presence of BG 45S5 might influence the fate of these cells earlier than polymeric materials.

\subsection{Immunocytochemistry}

As for the assessment of osteopontin expression of rat bone marrow cells cultured in the presence of PaII and
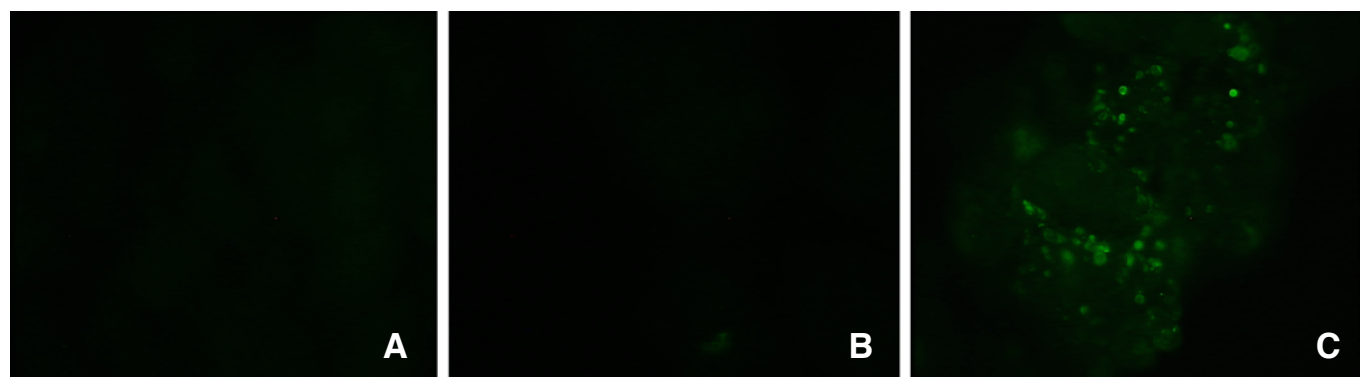

Fig. 15. Immunocytochemistry for osteopontin in PaII particles after 14 days of culture. (A) PaII particles' autofluorescence; (B) control, PaII particles (without cells) treated with anti-osteopontin antibody; (C) PaII particles cultured with rat bone marrow cells treated with osteopontin antibody. For both conditionspresence and absence of dexamethasone-(A), (B), and (C) were identical; for this reason, only one image per condition is shown. 

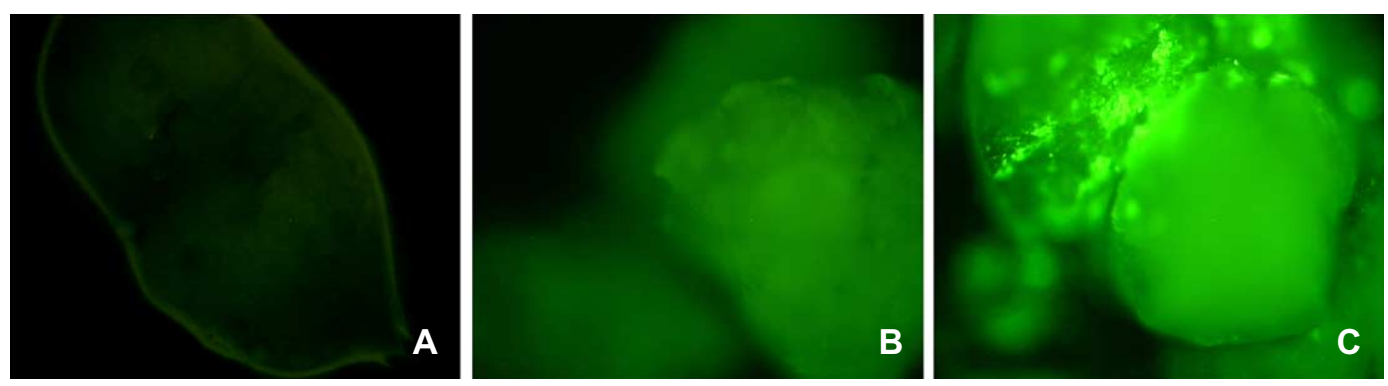

Fig. 16. Immunocytochemistry for osteopontin in PaII-30\% BG particles after 14 days of culture. (A) Control, PaII/BG particles' autofluorescence; (B) control, PaII/BG particles (without cells) with anti-osteopontin antibody; (C) PaII/BG particles cultured with rat bone marrow cells treated with osteopontin antibody. For both conditions-presence and absence of dexamethasone-(A), (B), and (C) were identical; for this reason, only one image per condition is shown.

PaII-30\% BG particles, with or without dexamethasone supplemented to the culture medium, the results are observed in Figs. 15 and 16.

The results for PaII particles after 14 days in culture with rat bone marrow cells clearly show that osteopontin is being expressed, which indicates that cells have adhered to the materials and that the cells were being able to express bone markers. Another interesting result it that we found no differences in osteopontin detection between cultures with and without dexamethasone. It could be speculated that this result might be due to the influence of the materials on the rat bone marrow cells' fate, which would lead to a commitment of the cells to the osteogenic lineage regardless of the presence of dexamethasone. However, to confirm this hypothesis, further studies regarding other osteogenic markers, namely osteonectin, osteocalcin, collagen I, and runx-2/cbfa-1 expression, must be performed.

As for PaII-30\% BG particles, although both their autofluorescence and control show some staining, which is due to the presence of BG 45S5, there is a considerable difference in the fluorescence of the particles cultured with rat bone marrow cells, allowing to state that osteopontin is being expressed. As already seen for PaII particles, again no differences were found between culture conditions (absence or presence of dexamethasone). For this type of particles, the presence of $\mathrm{BG} 45 \mathrm{~S} 5$ might play a role in cell differentiation, since it is known from the literature $[28,29]$ that this type of bioactive glass is capable of enhancing cell differentiation. However, further studies must be performed to assess the validity of this hypothesis.

\section{Conclusions}

It is believed that there is a correlation between the in vitro bioactivity of a material and its ability to bond to bone. In this way, immersion of the materials in a fluid simulating body ionic composition and the eventual formation of a calcium phosphate layer at the surface of the materials denote a possible in vivo bioactive behaviour. In this work, both polymer (PaII) particles and composite (PaII-30\% $\mathrm{BG})$ particles were shown to form in vitro an apatite-like
Ca-P layer at their surface, which was analysed and shown to have a different nature in both materials.

Short-term cytotoxicity testing shows that cells cultured with both polymer and composite leachables remain clearly viable. Preliminary studies on the ability of these particles to support attachment and growth of undifferentiated rat bone marrow cells have shown that cells do adhere to the materials, and that they express osteopontin both in the presence or absence of dexamethasone in the culture medium.

These results constitute the basis of further studies involving these materials and primary cultures of bone marrow cells, both in standard cell culture conditions and in an HARV (high aspect ratio vessel) bioreactor, where other osteoblastic markers will be evaluated both by biochemical tests and gene expression profiles, in order to establish differences between both conditions. The aim is to move to use these particles as carriers for cells, forming tissue engineering constructs in appropriate bioreactor culture conditions.

\section{Acknowledgements}

G.A. Silva is recipient of a grant from the Portuguese Foundation for Science and Technology (FCT) (reference SFRH/BD/4648/2001).

This work was partially supported by FCT through funds from the POCTI and/or FEDER programmes.

The authors acknowledge António Salgado for valuable discussions concerning this work.

\section{References}

[1] C. Knabe, R. Gildenhaar, G. Berger, W. Ostapowicz, R. Fitzner, R.J. Radlanski, U. Gross, Biomaterials 18 (1997) 1339.

[2] R.L. Reis, A.M. Cunha, P.S. Allan, M.J. Bevis, Advances in Polymer Technology 16 (1997) 263.

[3] R.L. Reis, A.M. Cunha, M.J. Bevis, Modern Plastics 76 (1999) 73.

[4] M.J. Yaszemski, R.G. Payne, W.C. Hayes, R. Langer, A.G. Mikos, Biomaterials 17 (1996) 175.

[5] H.S. Azevedo, F.M. Gama, R.L. Reis, Biomacromolecules 4 (2003) 1703. 
[6] E. Cenni, G. Ciapetti, D. Granchi, C.R. Arcioloa, L. Savarino, S. Stea, L. Montanaro, A. Pizzoferrato, Toxicology in Vitro 13 (1999) 801.

[7] C.J. Kirkpatrick, V. Krump-Konvalinkova, R.E. Unger, F. Bittinger, M. Otto, K. Peters, Biomolecular Engineering 19 (2002) 211.

[8] A.P. Marques, R.L. Reis, J.A. Hunt, Biomaterials 23 (2002) 1471.

[9] I.B. Leonor, R.L. Reis, Journal of Materials Science. Materials in Medicine 14 (2003) 435.

[10] A.L. Oliveira, C. Elvira, R.L. Reis, B. Vazquez, J. San Roman, Journal of Materials Science. Materials in Medicine 10 (1999) 827.

[11] A.L. Oliveira, P.B. Malafaya, R.L. Reis, Biomaterials 24 (2003) 2575.

[12] G.A. Silva, F.J. Costa, O.P. Coutinho, S. Radin, P. Ducheyne, R.L. Reis, Journal of Biomedical Materials Research 70A (2004) 442.

[13] I.B. Leonor, A. Ito, K. Onuma, N. Kanzaki, R.L. Reis, Biomaterials 24 (2003) 579.

[14] A. Schroeder, G. Francz, A. Bruinink, R. Hauert, J. Mayer, E. Wintermantel, Biomaterials 21 (2000) 449.

[15] H. Ohgushi, M. Okumura, K. Inoue, S. Tamai, S. Tabata, Y. Dohi, Materials Science Engineering. C C1 (1994) 139.

[16] M.E. Gomes, V.I. Sikavitsas, E. Behravesh, R.L. Reis, A.G. Mikos, Journal of Biomedical Materials Research Part A 67A (2003) 87.

[17] C. Maniatopoulos, J. Sodek, A.H. Melcher, Cell Tissue Research 254 (1988) 317.
[18] A. Rosa, M.M. Belotia, R. van Noortb, Dental Materials 19 (2003) 768.

[19] T. Kokubo, H. Kushitani, S. Sakka, T. Kitsugi, T. Yamamuro, Journal of Biomedical Materials Research 24 (1990) 721.

[20] J. Zeltinger, J.K. Sherwood, D.A. Graham, R. Mueller, L.G. Griffith, Tissue Engineering 7 (2001) 557.

[21] T.F. Slater, B. Sawyer, U. Strauli, Biochimica et Biophysica Acta 77 (1963) 383

[22] T. Mosmann, Journal of Immunological Methods 65 (1983) 55

[23] G.A. Silva, C.M. Vaz, O.P. Coutinho, A.M. Cunha, R.L. Reis, Journal of Materials Science. Materials in Medicine 14 (2003) 1055.

[24] P.K. Smith, Analytical Biochemistry 150 (1985) 76.

[25] A.J. Salgado, M.E. Gomes, A. Chou, O.P. Coutinho, R.L. Reis, D.W. Hutmacher, Materials Science Engineering. C, Biomimetic and Supramolecular Systems 20 (2002) 27.

[26] M. Tanahashi, T. Matsuda, Journal of Biomedical Materials Research 34 (1997) 305.

[27] P. Li, K. Nakanishi, T. Kokubo, K. de Groot, Biomaterials 14 (1993) 963.

[28] P. Ducheyne, A. El-Ghannam, I.M. Shapiro, Journal of Cellular Biochemistry 56 (1994) 162.

[29] A. El-Ghannam, I.M. Shapiro, P. Ducheyne, Journal of Biomedical Materials Research 29 (1995) 359. 